University of Texas at El Paso

ScholarWorks@UTEP

$10-2012$

\title{
How to Define Relative Approximation Error of an Interval Estimate: A Proposal
}

Vladik Kreinovich

The University of Texas at El Paso, vladik@utep.edu

Follow this and additional works at: https://scholarworks.utep.edu/cs_techrep

Part of the Applied Mathematics Commons

Comments:

Technical Report: UTEP-CS-12-37

Published in Applied Mathematical Sciences, 2013, Vol. 7, No. 5, pp. 211-216.

\section{Recommended Citation}

Kreinovich, Vladik, "How to Define Relative Approximation Error of an Interval Estimate: A Proposal" (2012). Departmental Technical Reports (CS). 726.

https://scholarworks.utep.edu/cs_techrep/726

This Article is brought to you for free and open access by the Computer Science at ScholarWorks@UTEP. It has been accepted for inclusion in Departmental Technical Reports (CS) by an authorized administrator of ScholarWorks@UTEP.For more information, please contact Iweber@utep.edu. 


\title{
How to Define Relative Approximation Error of an Interval Estimate: A Proposal
}

\author{
Vladik Kreinovich \\ Department of Computer Science \\ University of Texas at El Paso \\ El Paso, TX 79968, USA \\ vladik@utep.edu
}

\begin{abstract}
The traditional definition of a relative approximation error of an estimate $\widetilde{x}$ as the ratio $\frac{|\widetilde{x}-x|}{|x|}$ does not work when the actual value $x$ is 0 . To avoid this problem, we propose a new definition $\frac{|\widetilde{x}-x|}{|\widetilde{x}|}$. We show how this definition can be naturally extended to the case when instead of a numerical estimate $\widetilde{x}$, we have an interval estimate $[\underline{x}, \bar{x}]$, i.e., an interval that is guaranteed to contain the actual (unknown) value $x$.
\end{abstract}

How relative error is usually defined: reminder. There are two main ways to gauge how accurate is an estimate $\widetilde{x}$ of the actual (not precisely know) value $x$ (see, e.g., [3]):

- the absolute approximation error defined as $|\widetilde{x}-x|$, and

- the relative approximation error which is usually defined as ratio of the absolute error and the actual value:

$$
\frac{|\widetilde{x}-x|}{|x|}
$$

Since the actual value $x$ is now known exactly, we cannot compute the exact values of the corresponding errors. Often, however, we know the upper bounds on one of these errors (or or both of them). Such an upper bound serves as a description of approximation accuracy: we can say that we have an approximation with an accuracy of \pm 0.1 , or with an accuracy of $5 \%$.

The problem with the usual definition of a relative error. When the actual value is positive or negative, relative error makes perfect sense: the smaller the relative error, the close the estimate $\widetilde{x}$ to the actual value $x$. However, in a 
frequent situation when the actual value is 0 , relative error does not make sense: no matter how close is our estimate $\widetilde{x}$ to 0 , the relative error is infinite.

It is therefore desirable to come up with a more adequate relative description of approximation error.

A new definition of the relative error: a suggestion. We propose, when defining relative error, to divide the absolute error not by the actual (not precisely known) value $x$, but rather by the approximate (known) value $\widetilde{x}$. In other words, we propose the following new definition of the relative approximation error:

$$
r \stackrel{\text { def }}{=} \frac{|\widetilde{x}-x|}{|\widetilde{x}|}
$$

An argument in favor of the new definition. With this new definition, if we know the approximate estimate $\widetilde{x}$, and we know the upper bound $\delta$ on the relative error $r$, we can conclude that the actual value $x$ is somewhere between $\widetilde{x}-\delta \cdot \widetilde{x}$ and $\widetilde{x}+\delta \cdot \widetilde{x}$.

For example, if we have an approximate value 2 , and we know that the relative error is no more than $5 \%$, then in this new definition this would simply mean that the actual value is somewhere between

$$
2-0.05 \cdot 2=1.9 \text { and } 2+0.05 \cdot 2=2.1 \text {. }
$$

In contrast, in the traditional definition, it is not straightforward to come up with an interval of possible values.

How to extend this definition to interval estimates? Often, instead of a numerical estimate $\widetilde{x}$, we have an interval estimate $[\underline{x}, \bar{x}]$, i.e., an interval $[\underline{x}, \bar{x}]$ which is guaranteed to contain the actual (unknown) value $x$; see, e.g., $[1,2,3]$. How can we define relative accuracy of this interval estimate?

Discussion. In principle, we can select an arbitrary value $\widetilde{x}$ within this interval. For each selection, we get different values of a relative error depending on which of the values $x$ from the interval $[\underline{x}, \bar{x}]$ is the actual value of the corresponding quantity.

Thus, for this selection $\widetilde{x}$, the only guarantee that we can make about the relative error is that it does not exceed the maximum value

$$
r(\widetilde{x}) \stackrel{\text { def }}{=} \max \left\{\frac{|\widetilde{x}-x|}{|\widetilde{x}|}: \underline{x} \leq x \leq \bar{x}\right\} .
$$

Different values $\widetilde{x}$ from the interval $[\underline{x}, \bar{x}]$ lead, in general, to different values of $r(\widetilde{x})$. As the relative approximation error corresponding to the interval $[\underline{x}, \bar{x}]$, it is reasonable to select the smallest of the corresponding values $r(\widetilde{x})$. In other words, we arrive at the following definition: 
Definition. The relative approximation error $r$ of an interval estimate $[\underline{x}, \bar{x}]$ is defined as

$$
r \stackrel{\text { def }}{=} \min _{\tilde{x} \in[\underline{x}, \bar{x}]} r(\widetilde{x}),
$$

where

$$
r(\widetilde{x}) \stackrel{\text { def }}{=} \max _{x \in[\underline{x}, \bar{x}]} \frac{|\widetilde{x}-x|}{|\widetilde{x}|} .
$$

Comment. In other words, the relative approximation error is defined as

$$
r=\min _{\tilde{x} \in[\underline{x}, \bar{x}]} \max _{x \in[\underline{x}, \bar{x}]} \frac{|\widetilde{x}-x|}{|\widetilde{x}|} .
$$

How to actually compute this relative approximation error? The above definition may be reasonable, but it does not lead to straightforward computations. These computations can become easier if we use the following equivalent definition:

\section{Proposition.}

- When $\underline{x}$ and $\bar{x}$ have the same sign, then

$$
r=\frac{\bar{x}-\underline{x}}{|\underline{x}+\bar{x}|} .
$$

- When $\underline{x}$ and $\bar{x}$ are of different signs, i.e., when $\underline{x}<0<\bar{x}$ we have

$$
r=\frac{\bar{x}-\underline{x}}{\max (|\underline{x}|, \bar{x})} .
$$

Comment. When $\underline{x}$ and $\bar{x}$ have the same sign, $r$ can be equivalently defined as the ratio of the interval's radius $\frac{\bar{x}-\underline{x}}{2}$ to the absolute value of the interval's midpoint $\frac{x+\bar{x}}{2}$.

\section{Proof.}

$1^{\circ}$. Since the expression $\frac{|\widetilde{x}-x|}{|\widetilde{x}|}$ is a convex function of $x$, its maximum is attained at one of the endpoints, i.e.,

$$
r(\widetilde{x})=\max \left(\frac{|\widetilde{x}-\underline{x}|}{|\widetilde{x}|}, \frac{|\widetilde{x}-\bar{x}|}{|\widetilde{x}|}\right) .
$$


$2^{\circ}$. When $0 \leq \underline{x}<\bar{x}$, this formula becomes

$$
r(\widetilde{x})=\max \left(\frac{\widetilde{x}-\underline{x}}{\widetilde{x}}, \frac{\bar{x}-\widetilde{x}}{\widetilde{x}}\right),
$$

i.e.,

$$
r(\widetilde{x})=\max \left(1-\frac{x}{\widetilde{x}}, \frac{\bar{x}}{\widetilde{x}}-1\right)
$$

The first of the two maximized functions is increasing in $\widetilde{x}$, the second is decreasing in $\widetilde{x}$, so the minimum is attained when these two functions have the same value, i.e., when

$$
1-\frac{x}{\widetilde{x}}=\frac{\bar{x}}{\widetilde{x}}-1
$$

This equality leads to

$$
\widetilde{x}=\frac{x+\bar{x}}{2}
$$

In this case, $r=r(\widetilde{x})$ becomes the desired value

$$
\frac{\bar{x}-\underline{x}}{\underline{x}+\bar{x}}
$$

$3^{\circ}$. The case when $\underline{x}<\bar{x} \leq 0$ can be proven similarly.

$4^{\circ}$. Let us now consider the remaining case, when $\underline{x}<0<\bar{x}$.

$4.1^{\circ}$. When $\underline{x}<0<\bar{x}$ and $\widetilde{x} \geq 0$, we get

$$
r(\widetilde{x})=\max \left(\frac{\widetilde{x}+|\underline{x}|}{\widetilde{x}}, \frac{\bar{x}-\widetilde{x}}{\widetilde{x}}\right),
$$

i.e.,

$$
r(\widetilde{x})=\max \left(1+\frac{|\underline{x}|}{\widetilde{x}}, \frac{\bar{x}}{\widetilde{x}}-1\right) .
$$

Both maximized functions are decreasing with $\widetilde{x}$, so the minimum is attained when $\widetilde{x}$ takes the largest possible value, i.e., when $\widetilde{x}=\bar{x}$. For this value $\widetilde{x}$, the corresponding expression for $r(\widetilde{x})$ takes the form

$$
r(\bar{x})=\max \left(1+\frac{|\underline{x}|}{\bar{x}}, \frac{\bar{x}}{\bar{x}}-1\right)=\max \left(\frac{\bar{x}+|\underline{x}|}{\bar{x}}, 0\right),
$$

or, equivalently,

$$
r(\bar{x})=\max \left(\frac{\bar{x}-\underline{x}}{\bar{x}}, 0\right) .
$$

Since the first of the two maximized expressions is always non-negative, we get

$$
r(\bar{x})=\frac{\bar{x}-\underline{x}}{\bar{x}} .
$$


4.2 $2^{\circ}$ Similarly, when $\underline{x}<0<\bar{x}$ and $\widetilde{x}<0$, the smallest possible value of $r(\widetilde{x})$ is attained when $\widetilde{x}=\underline{x}$, in which case

$$
r(\underline{x})=\frac{\bar{x}-\underline{x}}{|\underline{x}|} .
$$

$4.3^{\circ}$. In general, for ]underlinex $<0<\bar{x}$, the smallest possible value $r$ is thus the smallest of the values $r(\bar{x})$ and $r(\underline{x})$ corresponding to two possible cases $\widetilde{x} \geq 0$ and $\widetilde{x}<0$. In other words,

$$
r=\min \left(\frac{\bar{x}-\underline{x}}{\bar{x}}, \frac{\bar{x}-\underline{x}}{|\bar{x}|}\right) .
$$

These two fractions have the same numerator, so the smallest value corresponds to the largest denominator:

$$
r=\frac{\bar{x}-\underline{x}}{\min (|\underline{x}|, \bar{x})} .
$$

The proposition is proven.

Acknowledgments. This work was supported in part by the National Science Foundation grants HRD-0734825 and HRD-1242122 (Cyber-ShARE Center of Excellence) and DUE-0926721, by Grant 1 T36 GM078000-01 from the National Institutes of Health, and by a grant on F-transforms from the Office of Naval Research.

The author is thankful to Siegfried Rump for his inspiration.

\section{References}

[1] L. Jaulin, M. Kieffer, O. Didrit, and E. Walter, Applied Interval Analysis, with Examples in Parameter and State Estimation, Robust Control and Robotics, Springer-Verlag, London, 2001.

[2] R. E. Moore, R. B. Kearfott, and M. J. Cloud, Introduction to Interval Analysis, SIAM Press, Philadelphia, Pennsylvania, 2009.

[3] S. Rabinovich, Measurement Errors and Uncertainties: Theory and Practice, Springer-Verlag, New York, 2005. 